\title{
Kapitza resistance cooling of single crystal (111) niobium for superconducting rf cavities
}

\author{
Jay Amrit ${ }^{1, *}$ and Claire Z. Antoine ${ }^{2}$ \\ ${ }^{1}$ LIMSI-CNRS, Université Paris-Sud 11, BP 133, F-91403, Orsay, France \\ ${ }^{2}$ CEA, Irfu, SACM, Centre de Saclay, F-91191 Gif-sur-Yvette, France
}

(Received 4 December 2009; published 18 February 2010)

\begin{abstract}
The use of large grains or single crystal niobium to improve the $Q$ factor of superconducting rf cavities for particle accelerators, is presently under study. Heat extraction which plays a decisive role in the thermomagnetic stability of these devices depends on the thermal conductivity of niobium $K$ and the thermal boundary (Kapitza) resistance $R_{K}$ at the niobium/superfluid helium interface. Here we present the first measurements of $R_{K}$ performed between 1.5-2.1 K with single crystal (111) niobium, having two different surface morphologies, namely, a surface with a damage layer and a chemically polished surface. The thermal conductivity of the single crystal $\mathrm{Nb}$ samples is also simultaneously determined. For monocrystalline niobium we demonstrate that $R_{K}$ is an increasing primary limiting factor with temperature, contrary to the behavior found for polycrystalline cavities. The present investigation reveals for the first time that the presence of impurities (metallic particles and oxygen) within the damage layer leads to a stronger $R_{K}$, although the effective heat exchange area to the superfluid is increased. We further show the importance of dislocations in the thermal conductivity of monocrystalline niobium.
\end{abstract}

DOI: 10.1103/PhysRevSTAB.13.023201

PACS numbers: 85.25. $-\mathrm{j}, 68.65 .-\mathrm{k}, 44.10 .+\mathrm{i}$

\section{INTRODUCTION}

The superconducting rf (SRF) cavity quality factor is expressed as $Q=\omega U / P$, where $U$ is the stored energy and $(P / \omega)$ the power loss in the inner walls in one rf radian. At high fields $(~ \gtrsim 25 \mathrm{MV} / \mathrm{m}$ ), some dissipation occurs that leads to a dramatic decrease of the $Q$ factor in monocrystalline as well as polycrystalline cavities. Moderate baking $\left(48 \mathrm{~h}, 120^{\circ} \mathrm{C}\right)$ can reduce dissipation by a substantial amount. Nevertheless, a full understanding of the observed drop in the $Q$ factor at high accelerating fields still remains a challenge in R\&D superconducting rf cavity technology. There are several evidences that the dissipation observed at high field has a magnetic origin and is related to the nonlinear behavior of the BCS term in the surface resistance. As $H_{\text {rf }}$ (rf magnetic field amplitude) approaches $H_{C}$ (superconductor critical field), both the normal electron density and the BCS surface resistance $R_{\mathrm{BCS}}$ increase due to the effect of current pair breaking on thermal activation which in turn increases heating, thus making $R_{\mathrm{BCS}}$ nonlinear in the high field. For type II superconductors like $\mathrm{Nb}$, in the clean limit (i.e. when the phonon mean-free path is greater than the coherence length) and at low frequency, the high field nonlinear correction increases exponentially with field and temperature, and can give rise to thermal runaway [1,2]. This model can, in particular, explain the hot spots observed on cavities where bundles of trapped vortices can produce localized dissipative regions from which heat spreads over several tens of $\mathrm{mm}$. The magnetic/vortex origin of some of the hot spots have been

\footnotetext{
*jay.amrit@limsi.fr
}

recently demonstrated, as well as the effect of baking on them [3]. At higher field some localized defects (not necessarily related to the previous "hot spots") will eventually become normal conducting. Here again it can be the source of a thermal runaway ("quench").

If the thermal dissipation is effectively transferred to the helium bath, the thermal runaway will be prevented or at least it will appear at a higher field. It is therefore of paramount importance to determine the thermal behavior of niobium and the source of limitations in the thermal transfer.

We recall that during functioning, Joule dissipation of power $P$ on the inner walls of the SRF cavity is due to the magnetic field interaction with electrons and it is defined by $P=\frac{1}{2 \mu_{o}^{2}} R_{s}(T) B^{2}$, where $\mu_{o}$ is the magnetic permeability, $R_{S}(T)$ is the residual electrical resistance of $\mathrm{Nb}$, and $B$ is the magnetic field (in Tesla). Evacuation of this heat depends on the thermal conductivity of niobium $K$ and the Kapitza conductance $R_{K}^{-1}$ between the outer cavity wall and the surrounding superfluid helium coolant. The thermal conductivity of niobium is generally monitored by controlling parameters like (i) the chemical purity [residual-resistivity ratio (RRR)] [4,5], (ii) the grain size anisotropy and thermal resistance at boundaries between grains [6] in the case of polycrystals, especially in the vicinity of the welding of two half cells, and (iii) the deformation the niobium samples have been subjected to [7] and the dislocation density in $\mathrm{Nb}$.

Extensive studies [7-11] have established that the thermal conductivity of single crystal niobium has a rather complex behavior: strong decreases of $K$ after the samples have been subjected to mechanical deformation. The work 
presented here brings highlight to the thermomechanical history of the sample as another plausible cause leading to a decrease of $K$.

On the other hand, the Kapitza resistance $R_{K}$ is an intrinsic thermal boundary resistance due to a poor transmission of phonons (heat carriers) at the niobium/liquid $\mathrm{He}$ interface. This transmission is (as we shall see later) barely due to acoustic properties of the bulk niobium and the superfluid, unlike for solid/solid interfaces. Experiments carried out on clean well-characterized surfaces have shown [12] that the major factors which contribute to the transmission arise from the solid/liquid He interface morphology at microscales/nanoscales, with the exact dependence on surface chemical purity and surface roughness yet to be established in a general theory. Hence, $R_{K}$ has to be measured for each sample.

About five years ago, the study of superconducting cavities from single crystal niobium was implemented with the objective to improve thermal stability $[13,14]$. Recent experimental investigations have shown improvements in the performance of these cavities at accelerating gradients up to $40 \mathrm{MV} / \mathrm{m}$ with only chemical etching. Usually, a similar performance cannot be reached on polycrystalline cavities unless they are electropolished, a surface treatment much more difficult to reproduce than chemical etching [15].

We present here the first measurement of the Kapitza resistance between niobium single crystals and superfluid helium from 1.5 to $2.1 \mathrm{~K}$ at vapor pressure. The thermal conductivity of the single crystal is also determined in a disklike configuration from our measurements. Two types of surfaces were studied, namely, surfaces possessing a "damage layer" prior to any treatment and surfaces which were chemically polished. We discuss the impact of these surface treatments on single crystal niobium superconducting cavity performance, uniquely from a thermal point of view. The results obtained here will help clarify and quantify the thermal improvements expected due to the use of single crystal niobium, compared to polycrystalline niobium.

\section{EXPERIMENTAL SETUP AND SAMPLES}

\section{A. Cell configuration and measuring technique}

The experimental cell configuration is shown in Fig. 1 and it is the same as in Ref. [5]. The cell is a superfluid helium leak-tight cavity with two identical study sample disks mounted on either sides of a cylindrical support made of stainless steel. A manganin wire heater and a carbon resistor, which serves as a thermometer, are installed inside the cell. The cell is placed in a superfluid helium- 4 bath. The cell cavity is also filled with superfluid helium via a $1.2 \mathrm{~m}$ long capillary. The He-bath temperature is maintained constant as a heat flux $\dot{q}$ is dissipated in liquid $\mathrm{He}$ inside the cell. Under steady state conditions a temperature jump $\Delta T_{T}$ is established between the He-bath temperature
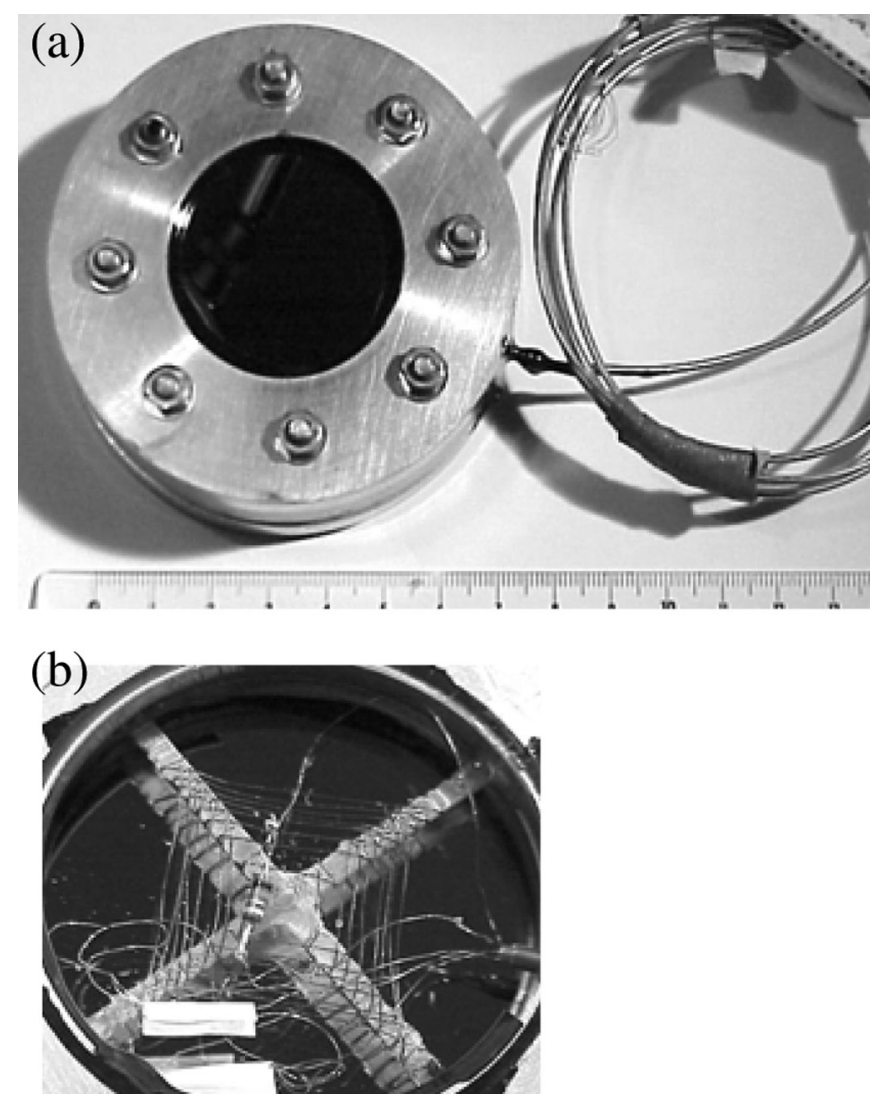

FIG. 1. Photo (a) is of the experimental cell. In (b) the inside of the cell is shown with the carbon thermometer at the center of the heater in the form of a web.

$T_{b}$ and the temperature inside the cavity $T_{i}$, for different heat fluxes. The temperature jump $\Delta T_{T}$ and the heat flux $\dot{q}$ are related as follows:

$$
\Delta T_{T}=\left(T_{i}-T_{b}\right)=\left(\frac{d}{2 K}+R_{K}\right) \dot{q},
$$

where $d$ is the sample disk thickness. The factor 2 in the equation is due to symmetry in the heat flux across each sample. By performing measurements on two pairs of samples, each pair having a different thickness but identical bulk properties and surface morphologies, the thermal conductivity and $R_{K}$ were determined. Details of the heat loss analysis, as described in Ref. [5], were carried out.

\section{B. Samples and their surface preparation}

The single crystal samples were cut out of a large grain niobium ingot of high purity ( RRR 300) manufactured by the company CBMM. A series of large grain sheets were sliced directly from the as cast ingot after its last electron beam melting. The study samples were cut by electroerosion, in the form of disks of $5 \mathrm{~cm}$ in diameter, from this series of slices which all originated from the same ingot. Precautions were taken to ensure that the crystallographic orientation of each of the study samples is identical. 
Two sample surfaces morphologies were studied, namely, surfaces as cut by electrical discharge machining $(\mathrm{EDM})$, with a "damage layer" and surfaces which were chemically polished.

The "damage layer" extends to a depth of $\sim 25 \mu \mathrm{m}$ into the sample surface. This surface is dark grey in color and has a velvetlike texture with a root mean square surface roughness $\sigma_{\text {rms }} \sim 6-7 \mu \mathrm{m}$. Also, the "damage layer" has an amorphous microcrystalline structure. It contains impurities (mainly oxygen, but also metallic impurities like $\mathrm{Cu}$ and $\mathrm{Zn}$ ) within the uppermost layer of $\sim 1 \mu \mathrm{m}$ as described in Ref. [16]. The impurity content is almost uniform along the surface and this impurity concentration decreases with depth from the crystal surface. The chemical etching process called buffered chemical polishing (BCP) was used to remove $\sim 30 \mu \mathrm{m}$ from the sample surface. The chemical
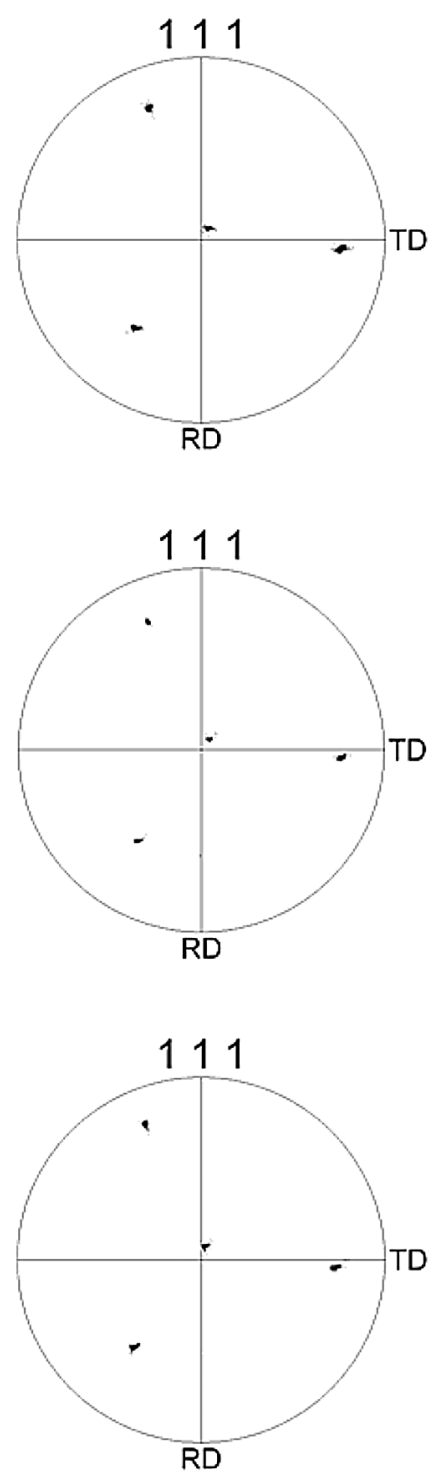

FIG. 2. Pole figures by EBSD at different locations on the $\mathrm{Nb}$ single crystal sample. polished samples were shiny, with a natural oxide layer of $\sim 5 \mathrm{~nm}$ thick on its surface. $\sigma_{\text {rms }}$ of this surface is reduced now by a factor of $\sim 5$. Prior to mounting the samples onto the experimental cell, they were placed in ultrapure ethanol and cleaned ultrasonically for $\sim 10$ minutes.

Electron backscattering pattern imaging was performed to confirm the crystallographic orientation throughout each of the samples. Pole figures obtained by electron backscattering diffusion (EBSD) for different locations on the sample are shown in Fig. 2. For all of the samples the heat flux was directed parallel to the (111) orientation; that is, the [111] crystal plane was in contact with superfluid helium.

\section{RESULTS}

A typical set of data recording conducted at $T_{b} \approx$ $1.817 \mathrm{~K}$ is shown in Fig. 3. In the temperature range of the experiment the relative sensitivity $d R /(R d T)$ of the carbon thermometer inside the cell lies between $(6.5-9.5) \times 10^{-4} \mathrm{mK}^{-1}$. The signals are amplified by a gain of 50. The combined effects of the $R_{K}$ and $(d / K)$ are clearly observable by the jumps in $T_{i}$ corresponding to changes in power dissipated inside the cell. The bath temperature is maintained under control using a calibrated LakeShore 200-GR $1000 \mathrm{Ge}$ resistor, to within less than $1 \mathrm{mK}$ throughout an entire experiment.

\section{A. Thermal conductivity}

The thermal conductivity $K$ is determined from measurements performed on the 2 and $4 \mathrm{~mm}$ chemically pol-

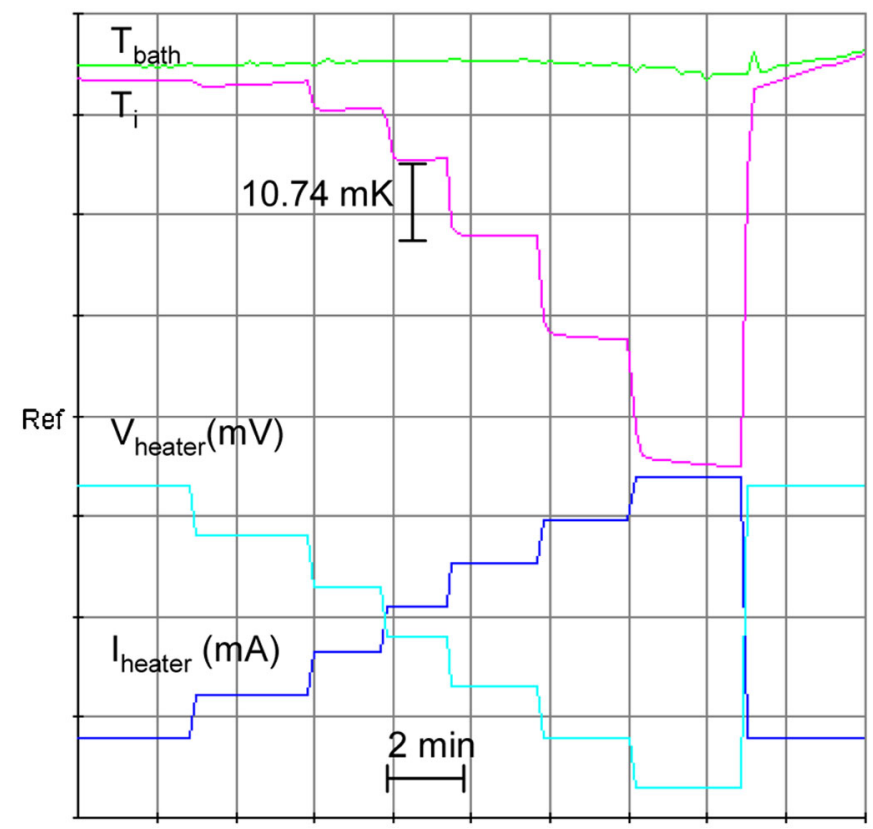

FIG. 3. (Color) Typical data recording with Datalogger ${ }^{\circledR}$, of the temperature evolution in the cell, for different heating powers. Measurement conducted at $T_{\text {bath }}=1.817 \mathrm{~K}$. The $T_{\text {bath }}$ stability is better than $1 \mathrm{mK}$. 


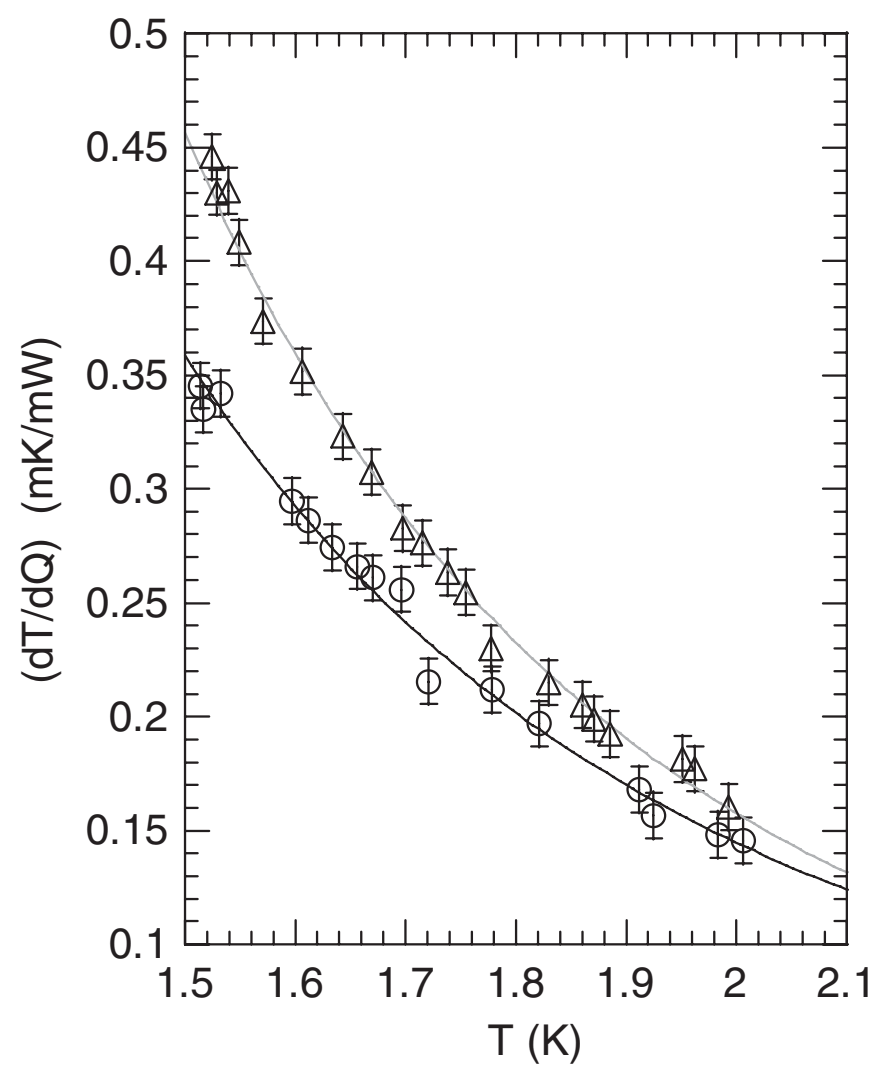

FIG. 4. Temperature evolution of the measured $(d T / d \dot{Q})$ for $2 \mathrm{~mm}$ (circles) and $4 \mathrm{~mm}$ (triangles) chemically polished single crystal. The lines through each set of data points are power law fits.

ished samples. The total temperature variation with heat flux $(d T / d \dot{Q})$ across these two samples [see Eq. (1)] is shown in Fig. 4. To determine $K$ we took $R_{K}$ to be the same for the two chemically polished samples. We justify this by recalling that the surface states, bulk chemical purity, and crystal orientations are identical for these samples. Using the power law fits to each set of data in Fig. 4, our thermal conductivity is determined as shown in Fig. 5 (squares) as a function of temperature.

To show the rather complex behavior of the thermal conductivity of our single crystal niobium, we indicate in Fig. 5 [curve $a$ ] the theoretical phonon thermal conductivity of an undeformed superconducting single niobium crystal, given by the Casimir equation [9] $K / T^{3}=$ $\left\{2310 R\left(2 \alpha^{2}+1\right) /\left(2 \alpha^{3}+1\right)\left(C_{v} / T^{3}\right)^{2 / 3}\right\}$, where $C_{v}=$ $8.57 \times 10^{-6} T^{3} \mathrm{~J} \mathrm{~cm}^{-3} \mathrm{~K}^{-4}$, the sample thickness $2 R=$ $0.2 \mathrm{~cm}$ and $\alpha=2.2$ is the ratio of the longitudinal $\left(v_{l}=\right.$ $4720 \mathrm{~m} / \mathrm{s})$ to transverse $\left(v_{t}=2140 \mathrm{~m} / \mathrm{s}\right)$ sound velocity. Curve $a$ in Fig. 5 corresponds only to the ballistic scattering of phonons by the sample boundaries; the electronic contribution to the thermal conductivity is not included. The discrepancy between the experimental data and curve $a$ is evidently large. From the kinetic gas model expression for the thermal conductivity $K=(1 / 3) C_{p} v_{D} l_{\text {eff }}$, where the specific heat $C_{p}=8.62 \times 10^{-6} T^{3} \mathrm{~J} \mathrm{~cm}^{-3} \mathrm{~K}^{-4}$ and the

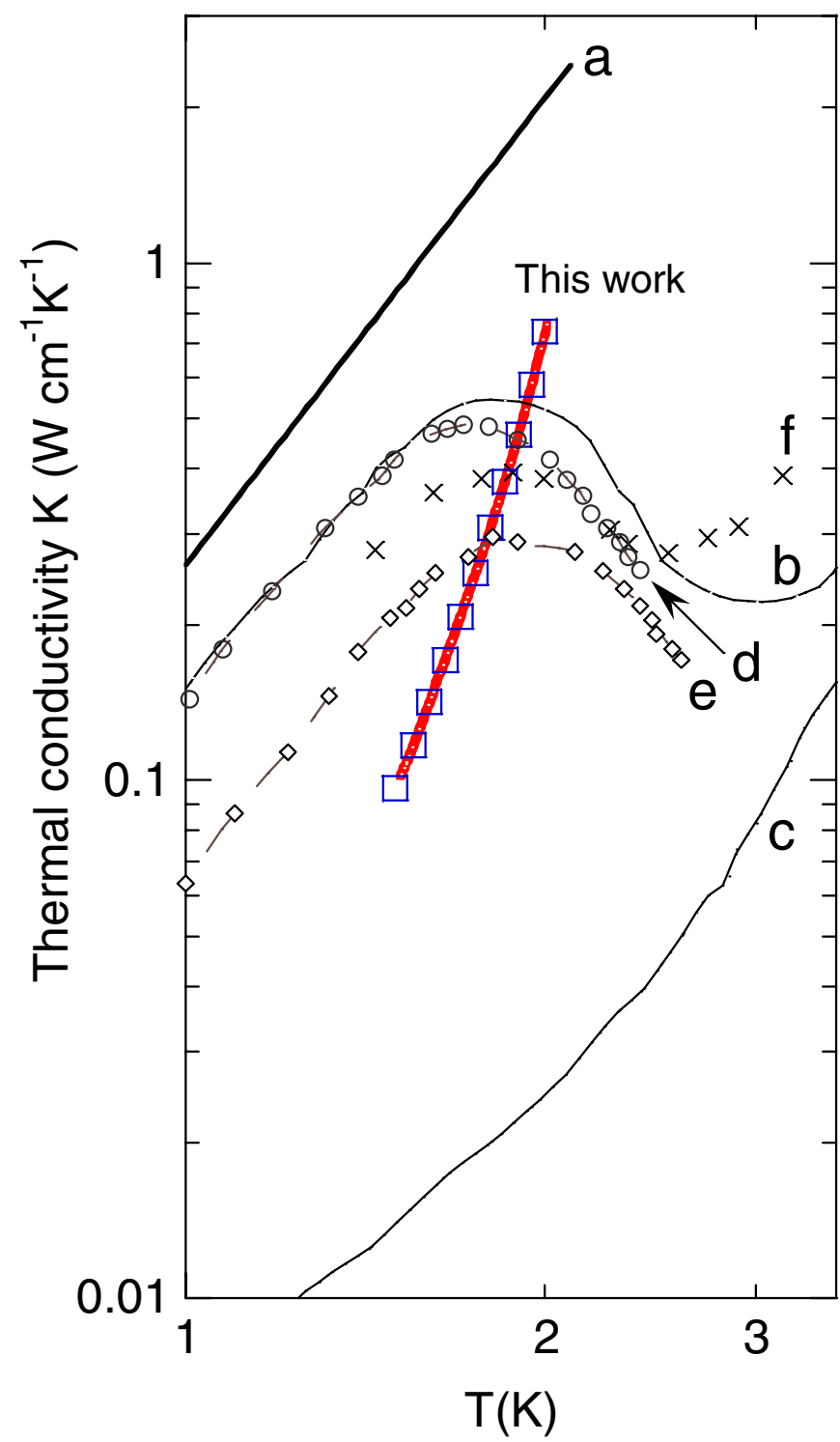

FIG. 5. (Color) Comparison of thermal conductivity of $\mathrm{Nb}$. Thermal conductivity measurements of our $\mathrm{Nb}$ (111) single crystal (squares). Curve $a$ represents Casimir scattering of phonons by boundaries (see text). Curves $b$ and $c$ correspond, respectively, to a single crystal $\mathrm{Nb}(\mathrm{RRR}=295)$ before and after undergoing a $14.7 \%$ deformation [8]. Data points labeled $d$ (circles) and $e$ (diamonds) are taken from Ref. [11] and they correspond, respectively, to undeformed single crystal $\mathrm{Nb}$ having RRR values of 196 and 135. Data points $f$ (crosses) are of polycrystalline $\mathrm{Nb}$ with $\mathrm{RRR}=400$ [17]. Curve fit to our data is given by $K=\left[\left(10 / T^{3}\right)+(3.05 \times\right.$ $\left.\left.10^{-6} N_{d}\right) / T^{2}\right]^{-1} \mathrm{~W} \mathrm{~cm}^{-1} \mathrm{~K}^{-1}$.

Debye velocity $v_{D} \approx 2.411 \times 10^{5} \mathrm{~cm} / \mathrm{s}$, we determined the phonon effective mean-free path $l_{\text {eff }}$ as shown in Fig. 6 . Expressing the phonon effective mean-free path as $l_{\text {eff }}=$ $\left(1 / l_{b}+1 / l_{i}\right)^{-1}$, we have that the mean-free paths of phonon in our single crystal are not limited by the sample surface boundaries $l_{b}$, but rather by $l_{i}$, an effective meanfree path due to internal phonon scattering mechanisms 


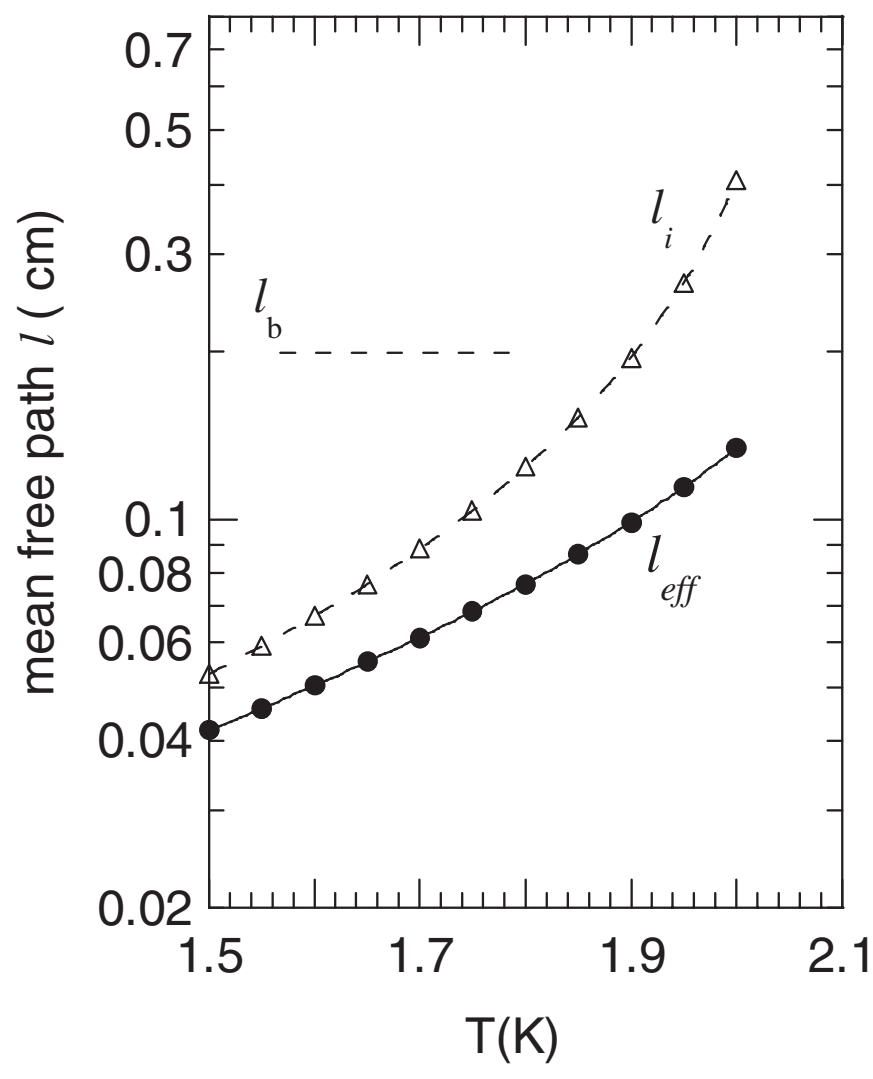

FIG. 6. The phonon effective mean-free $l_{\text {eff }}=\left(1 / l_{b}+1 / l_{i}\right)^{-1}$ is calculated from our thermal conductivity data. $l_{i}$ is the meanfree path associated with all mechanisms excepting boundary scattering, characterized by $l_{b}$.

[such as phonon-dislocation interactions and scattering of phonons by electrons even though $t=\left(T / T_{c}\right)<0.2$, where the critical temperature $\left.T_{c}=9.2 \mathrm{~K}\right]$. Therefore our experimentally determined $K$ values given in Fig. 5 (squares) do indeed correspond to the real thermal conductivity of our niobium (111) and it is not an effective geometry-dependent thermal conductivity. Generally, the walls of the SRF cavities are $\sim 3 \mathrm{~mm}$ thick and our experimental cell geometry is close to the real cavity configuration.

In the temperature range $1-2 \mathrm{~K}$, the thermal conductivity is strongly influenced by the degree of plastic deformation the monocrystalline niobium has been subjected to $[7,8]$. For comparison we note that the orders of magnitude of our data (squares) agree very satisfactorily with other measurements shown in Fig. 5 by curves $b, d$, and $e$ which correspond to data of "undeformed" niobium monocrystals having, respectively, RRR values of 250 [7], 196 [11], and 135 [11]. Our sample has a RRR of 300. Apparently, our thermal conductivity data does not show a "peak" in the thermal conductivity. The peak is related to the competition between the electronic and the phonon component of the thermal conduction, and to the crystalline state of the material. For niobium single crystals the peak generally begins at temperatures close to $\sim 1 \mathrm{~K}$ depending upon the degree of deformation and it attains a maximum at $\sim 1.8 \mathrm{~K}$ as discussed in Ref. [7]. The peak disappears for deformations $\gtrsim 10 \%$ as shown in Fig. 5 by curve $c$, which is due to a $14.7 \%$ deformation of the sample of curve $b$. The absence of the peak behavior is a characteristic signature of a $\mathrm{Nb}$ single crystal possessing a non-negligible distribution of dislocations. For completeness, we note that in polycrystalline niobium, the presence of the peak is monitored by the crystal grain size. This is so since the important scattering sites here are the boundaries of the crystal grains. In Fig. 5, the crosses (labeled $f$ ) represent typical data for polycrystalline niobium with grain sizes $\sim 2 \mathrm{~mm}$ and a RRR of 400 [17].

To interpret our measurements in Fig. 5 using models of thermal conductivity which take into account kink formation and motion on dislocations and phonon-kink interactions, is beyond the scope of this paper since the nature of the dislocations (screw, nonscrew, edge), dislocation arrangement (aligned, tangled), and dislocation densities present in our sample are required. Instead we do a simple analysis of our experimental data using a model which considers the scattering of phonons by randomly distributed dislocations. In this model, the lattice thermal resistivity associated with the phonon-dislocation interaction is calculated to be $R_{d}=3.05 \times$ $10^{-9} N_{d} / T^{2} \mathrm{~cm}^{3} \mathrm{~K}^{3} / \mathrm{W}$, where $N_{d}$ is the dislocation density per unit area. The lattice thermal conductivity is then given by $K_{l}=\left[\left(K_{b}\right)^{-1}+R_{d}\right]^{-1}$, where $\left(K_{b}\right)^{-1}=$ $3 /\left(C_{p} v_{D} l_{b}\right) \approx 10 / T^{3} \mathrm{~cm} \mathrm{~K} / \mathrm{W}$ is the thermal resistivity due to boundary scattering. In fitting our data with $K_{l}$ we took $N_{d}$ to be an adjustable parameter. The best fit to our data shown in Fig. 5 is obtained for $10^{8} \leqq N_{d} \lesssim$ $5 \times 10^{9} \mathrm{~cm}^{-2}$. These orders of magnitude of $N_{d}$ are in excellent agreement with those found for niobium monocrystals having undergone $<15 \%$ deformations $[7,18]$. Our analysis clearly illustrates the important role of dislocations for monocrystalline niobium in the temperature range 1.5-2.5 K. For completeness, we add that undeformed $\mathrm{Nb}$ single crystals generally have dislocations densities of $10^{5}-10^{7} \mathrm{~cm}^{-2}$ after a heat treatment [11].

We now address the question concerning the origin of dislocations in our sample. Our samples were not mechanically deformed and the EDM cutting is known to affect only a few microns of the surface. Since the main component of the thermal conductivity is bulklike, we suppose that the absence of a peak is related to the thermal stress that occurred during cooling of the ingot. Indeed, during the crystal solidification process the ingot is subjected to typical temperature changes of the order of $\Delta T \sim 2500 \mathrm{~K}$. Since the melting temperature of niobium is $T_{m} \sim 2740 \mathrm{~K}$, the samples may contain and store dislocations at room temperatures. Using an average value of the thermal coefficient of $(d \ell / d T) / \ell \approx 10^{-5} \mathrm{~K}^{-1}$, we have thermally induced deformations of the order of 2\%-3\%. Recent measurements [19] have shown that the thermal conduc- 
tivity peak is suppressed for deformations as small as $\sim 3 \%$.

Clearly, the presence of the peak leads to higher thermal conductivities and therefore is advantageous from a thermal point of view for SRF cavities. Moderate heating $\left(600-800^{\circ} \mathrm{C}\right)$ is liable to improve the thermal conductivity.

\section{B. Kapitza resistance}

Figure 7 shows our measurements of $R_{K}$ conducted on single crystal $\mathrm{Nb}$ samples in which the heat flux is parallel to the [111] crystal orientation direction. Two different surface states of these samples are studied. Data on the chemically polished samples were obtained on samples having thicknesses of $2 \mathrm{~mm}$ (full triangles) and $4 \mathrm{~mm}$ (full dots). The samples with the damage layer (full diamonds) were $2 \mathrm{~mm}$ thick. The power laws fitting the chemically polished and the damage layer sample data in Fig. 7 are, respectively, $R_{K}=8.479 \times T^{-2.482} \mathrm{~cm}^{2} \mathrm{~K} / \mathrm{W}$ and $R_{K}=9.932 \times T^{-2.062} \mathrm{~cm}^{2} \mathrm{~K} / \mathrm{W}$. The measurements distinctly show that $R_{K}$ of the damage layer sample is $30 \%-40 \%$ greater than that of the chemically polished sample. This result, as discussed later, concords with observations made in a previous study [5] on polycrystalline niobium cavities which contained a high concentration of Ti at its surface.

The orders of magnitude of our $R_{K}$ measurements at the chemically polished niobium single crystal (111)/superfluid helium interface are comparable to $R_{K}$ values obtained on chemically polished polycrystalline

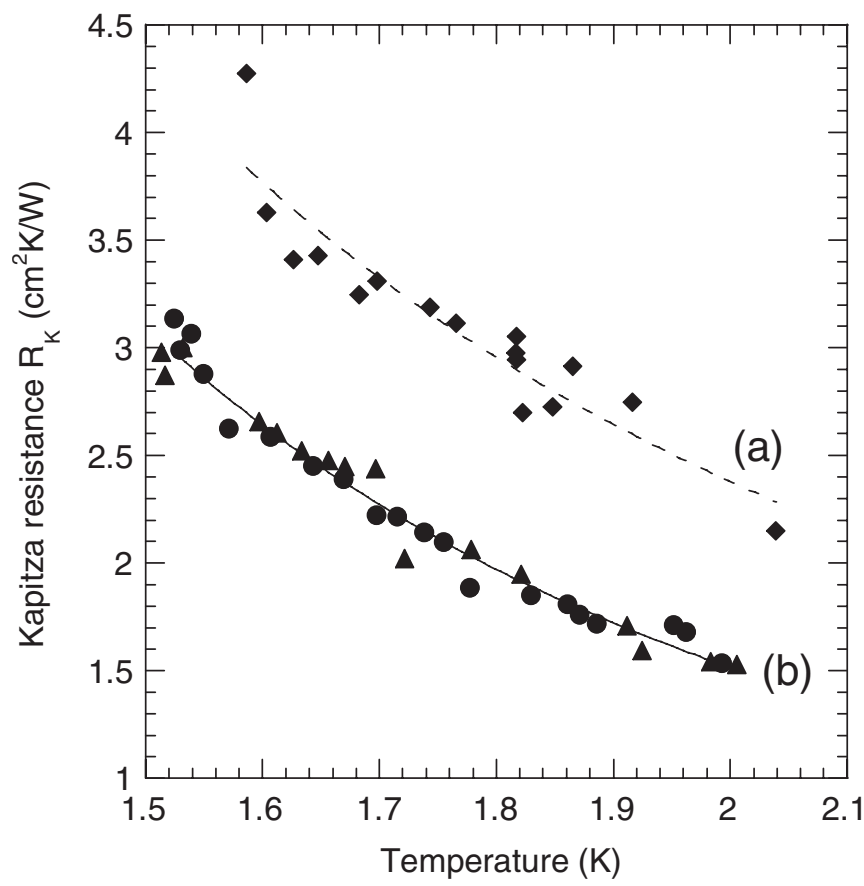

FIG. 7. $R_{K}$ measurements for (a) samples with a damage layer and (b) chemically polished samples. The dots and triangles correspond to samples of thickness 4 and $2 \mathrm{~mm}$, respectively. The lines through the data are power law fits given in the text. niobium samples having an RRR of 647, in Ref. [5]. We recall that the chemically polished polycrystalline niobium sample had a similar surface roughness as in the present study and its Kapitza resistance was found to be $R_{K}=$ $16.1 \times T^{-3.93} \mathrm{~cm}^{2} \mathrm{~K} / \mathrm{W}$. In the temperature range $1.5-$ $2 \mathrm{~K}$, the relative discrepancy in $R_{K}$ between the monocrystalline and the polycrystalline niobium attains a maximum of $\sim 30 \%$ at $\sim 2 \mathrm{~K}$.

We now examine the relative impact of $R_{K}$ and the thermal resistance $(d / 2 K)$ [see Eq. (1)] on the heat transport process. To show this, we plot the ratio $R_{K} /\left[R_{K}+\right.$ $(d / K)]$ as a function of temperature as shown in Fig. 8. In the working temperature range of SRF cavities (1.6-2 K), $R_{K}$ for monocrystalline niobium constitutes a gradually increasing percentage of the total thermal resistance to heat flowing out off the cavities. For example, at $T \sim$ $1.8 \mathrm{~K}, R_{K} /\left[R_{K}+(d / K)\right] \approx 75 \%$ for the chemically polished sample (triangles in Fig. 8). Consequently, $R_{K}$ is by far the dominant limiting factor compared to the thermal resistance $(d / 2 K)$ as the temperature is increased. However, the contrary is observed, as displayed by curve $c$ in Fig. 8, for the chemically polished polycrystalline niobium sample described above. This discrepancy is due to the combined effects of a stronger temperature dependency of $R_{K}$ and a smaller thermal conductivity of polycrystalline niobium, even though the RRR is a factor of $\sim 2$ bigger, compared to our monocrystalline niobium. In sum-

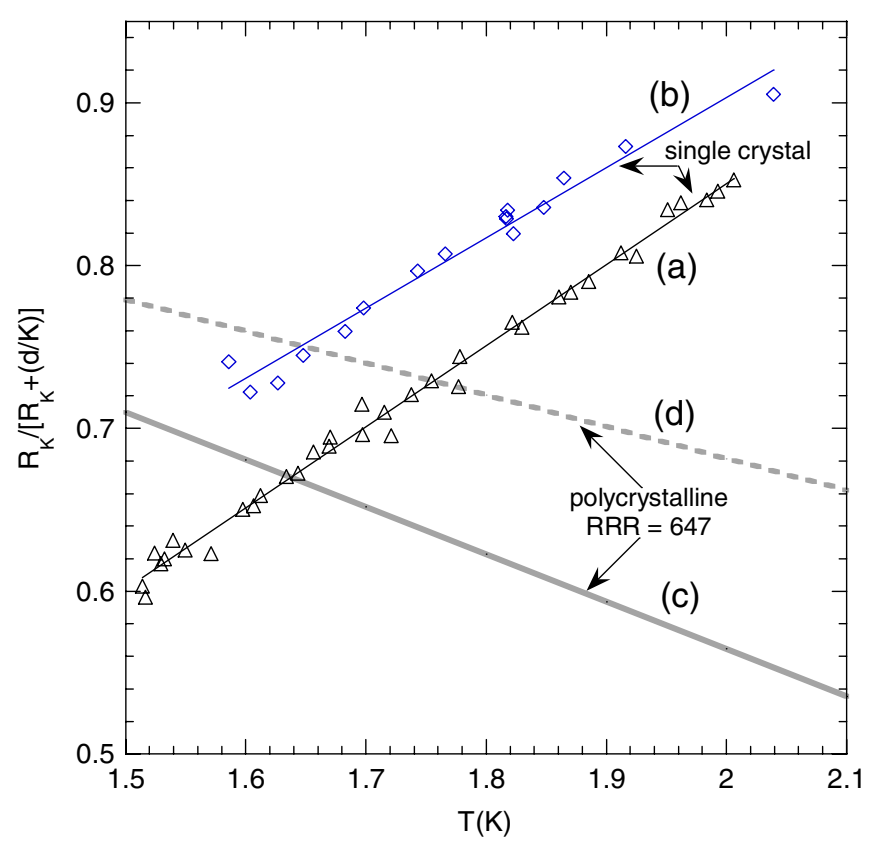

FIG. 8. (Color) Ratio of $R_{K}$ to the total thermal resistance $\left[R_{K}+\right.$ $(d / K)$ ] demonstrating the importance of $R_{K}$ as a function of $T$ for monocrystalline and polycrystalline $\mathrm{Nb}$. The diamonds and triangles correspond to our single crystal samples with a damage layer surface and a chemically polished surface, respectively. Curves $c$ and $d$ are for polycrystalline $\mathrm{Nb}$ samples which were, respectively, chemically polished and electropolished [5]. 
mary, $R_{K}$ constitutes the major impedance for heat evacuation from monocrystalline cavities.

On a more fundamental aspect of the Kapitza resistance, previous studies $[4,5]$ on polycrystalline niobium have shown that $R_{K}$ systematically decreases as the surface roughness is increased. Results of the present study, as seen in Fig. 7, tend to oppose this argument. Profilometry measurements indicate that the sample with the damage layer has peak heights and valleys of $\sim 25 \mu \mathrm{m}$. The effective surface of the damage layer sample is certainly greater than that of our chemically polished sample which has a surface roughness of approximately 5 times smaller than the damage layer sample. The present measurements therefore suggest that the phonon transmission at the interface is impaired due to the internal structure of the damage layer. Indeed, the latter has chemical impurities and structural disorder which extend to a depth of at least $20 \mu \mathrm{m}$ into the sample surface. In other words, the results reveal that surface quality, which includes chemical purity, structural order, and surface roughness, strongly impact $R_{K}$. Elemental analysis using energy dispersive spectroscopy in a scanning electron microscope, of the top $1 \mu \mathrm{m}$ layer of the monocrystalline $\mathrm{Nb}$ sample which was cut using the $\mathrm{EDM}$ wire technique as in this work, indicates that $\sim 10 \%$ of $\mathrm{Zn}$ and $14 \%$ of $\mathrm{Cu}$ particles are present in the $\mathrm{Nb}$ damage layer. Oxygen and hydrogen content are also increased [16]. The experimental $R_{K}$ results obtained here are coherent with other studies which showed that the presence of Ti impurity atoms in $\mathrm{Nb}$ within $\sim 5 \mu \mathrm{m}$ to the surface, leads to a greater $R_{K}$ (see Fig. 5 in Ref. [5]). Indeed, Ti was used in the 1990's as a getter material to purify niobium and to enhance its thermal conductivity when high purity niobium was not commercially available, as discussed in Ref. [20].

Now the thermomechanical history of $\mathrm{Nb}$ single crystals has a strong impact on the thermal conductivity and the Kapitza resistance. Since the surface of our "as is" EDMcut sample has an amorphous layer, it is more relevant to compare combined effects of $R_{K}$ and $K$ on heat transport in the case of a chemically polished $\mathrm{Nb}$ single crystal to that of a mechanically polished $\mathrm{Nb}$ single crystal. The latter has a larger surface roughness than a chemically polished sample. However, it is not evident that the effective heat transfer from the $\mathrm{Nb}$ crystal to the superfluid helium would be more efficient in the case of mechanically polished samples. This is so because mechanically polished $\mathrm{Nb}$ single crystal samples have a high density of dislocations [18] which decreases the thermal conductivity (see curve $c$ in Fig. 5). It is also possible that the presence of a high density of dislocations may modify the Kapitza resistance. This aspect requires new systematic experimental investigations. However, it is well known that a heat treatment (annealing) drastically reduces the dislocation density by a factor of 10-100 [11,18]. Consequently, we believe that a mechanically polished $\mathrm{Nb}$ single crystal which has under- gone a heat treatment shall have a better efficiency in thermal evacuation than a chemically polished $\mathrm{Nb}$ single crystal.

Challis [21] has shown that the Kapitza conductance is the heat (phonon) flux incident from the $\mathrm{Nb}$ onto the interface multiplied by an averaged transmission coefficient $\bar{\tau}_{\mathrm{Nb} \rightarrow \mathrm{He}}$ across the $\mathrm{Nb} / \mathrm{He}$ interface; that is, $R_{K}^{-1}=$ $(1 / 4) C_{p} v_{D} \bar{\tau}_{\mathrm{Nb} \rightarrow \mathrm{He}}$, where $C_{p}$ is the specific heat. Figure 9 shows the transmission calculated from our $R_{K}$ data of the two samples. The average transmission of the chemically polished sample and the sample with damaged layer are distinctly different as expected and show, respectively, the following temperature dependencies: $\bar{\tau} \sim 0.2 T^{-0.52}$ and $\bar{\tau} \sim 0.2 T^{-0.94}$. The theoretical transmission related to the acoustic mismatch of the bulk properties of each medium is estimated to be $\tau_{b}=\left(\rho_{\mathrm{He}} c_{\mathrm{He}} / \rho_{\mathrm{Nb}} c_{D}\right) \approx 0.00168$ [21], where we took $\rho_{\mathrm{He}}=0.145 \mathrm{~g} / \mathrm{cm}^{3}, \quad c_{\mathrm{He}}=2.4 \times$ $10^{4} \mathrm{~cm} / \mathrm{s}, \rho_{\mathrm{Nb}}=8.57 \mathrm{~g} / \mathrm{cm}^{3}$, and $v_{D}$ as above. In the determination of $\tau_{b}$ the interface surface is assumed to be atomically smooth on the length scale of the wavelengths of phonon in liquid $\mathrm{He}$, that is, of the order of 1$4 \mathrm{~nm}$. This condition certainly cannot be fulfilled in reality for our present samples. It now becomes evident that parallel channels of heat transmission, for which the exact mechanisms need to be identified, are clearly dominant. The average transmission must therefore correspond to

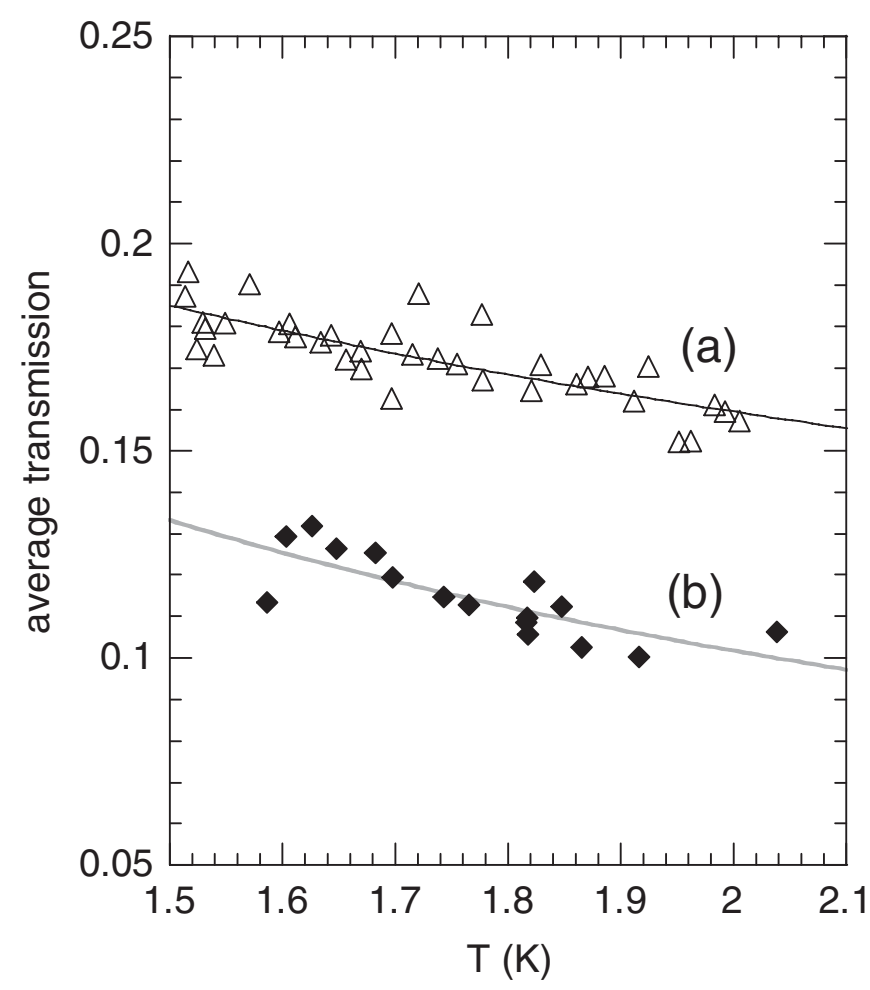

FIG. 9. Average transmission calculated using $R_{K}^{-1}=$ $(1 / 4) C_{p} v_{D} \bar{\tau}_{\mathrm{Nb} \rightarrow \mathrm{He}}$ for phonon heat flux parallel to the [111] orientation of single crystal $\mathrm{Nb}$ samples for (a) chemically polished surfaces and (b) surfaces with damage layer. The lines are fits through data given in the text. 
$\bar{\tau}_{\mathrm{Nb} \rightarrow \mathrm{He}}=\left(1 / \tau_{b}+1 / \tau_{s}\right)^{-1}$, where $\tau_{s}$ represents transmission from parallel channels. Our measurements show, as argued before, that this mechanism depends on the surface state since all of our samples have identical bulk purity and crystallographic orientation. Consequently, systematic experimental studies must be undertaken on single crystal niobium to ascertain the microscale/nanoscale surface morphology that minimizes $R_{K}$.

\section{COMMENTS AND CONCLUSIONS}

In summary, we present the first measurements of the Kapitza resistance between single crystal (111) niobium and liquid He between 1.5 and $2.1 \mathrm{~K}$. Two different surface states, namely a surface with a damage layer and a chemically polished surface, are studied. The thermal conductivity of the single crystal niobium is also determined.

Our experimental results show that surface chemical purity and structure play a decisive role in determining the magnitude of $R_{K}$. An increase in the effective surface area will not necessarily lead to a diminution of $R_{K}$. Contrary to expectations, $R_{K}$ of the sample with the damage layer is greater than that of the chemically polished sample, although the roughness (hence the developed surface area) is higher. This is a fundamental physical aspect which requires further investigation in order to understand the nature of the phonon-impurity diffusion mechanism close to the interface. We have also examined the role of dislocations in the thermal conductivity of monocrystalline niobium and have shown its importance. We recall that, in polycrystalline niobium, the grain boundaries impact the thermal conductivity in the $1.5-2.5 \mathrm{~K}$ range. We have analyzed the possibility of deformations generated by thermal stress during crystal growth of our samples. This is an important aspect which will help control/monitor the thermal conductivity of monocrystalline niobium. In light of SRF cavity performance, these findings imply that for a better efficiency in heat evacuation from the outer surfaces of cavities, nontreated single crystal niobium with a damage layer must be avoided. The degradation/modification of the outside surface shall certainly influence $R_{K}$ and therefore may lead to eventual modifications of the $Q$ factor of the cavity. $R_{K}$ values should also be considered when performing a comparative study of $Q$ drop among different experiments.

We have also demonstrated that for monocrystalline niobium having an RRR of $\sim 300, R_{K}$ constitutes the dominant thermal resistance over the entire working temperature range of the cavity. The Kapitza resistance is consequently a very important control parameter of the thermal stability and, therefore, of the efficiency of SRF cavities since the breakdown magnetic field $B_{b} \propto 1 / \sqrt{R_{K}}$ [2] in the limit of a high thermal conductivity.

We are now considering doing measurements of other single crystal orientations in order to determine if the present results are a general feature of the monocrystalline form of niobium or if it is strongly orientation dependent.

\section{ACKNOWLEDGMENTS}

We are indebted to the Fermi National Accelerator Laboratory in Illinois for kindly supplying the samples to one of us (C. A.). We also thank Thierry Baudin of the Laboratory of Physico-Chimie de l'Etat Solide, Orsay University, who performed the Electron Backscattering Diffusion measurements. One of us (J. A.) expresses his gratitude to student Jeremy Moriot and postdoctorate Venky Radhakrishnan for their interest and help at different stages of this work.

[1] P. Bauer, N. Solyak, G. L. Ciovati, G. Eremeev, A. Gurevich, L. Lilje, and B. Visentin, Physica C (Amsterdam) 441, 51 (2006).

[2] A. Gurevich, Physica C (Amsterdam) 441, 38 (2006).

[3] G. Ciovati and A. Gurevich, Phys. Rev. ST Accel. Beams 11, 122001 (2008).

[4] J. Amrit, C.Z. Antoine, M. X. François, and H. Safa, Adv. Cryog. Eng. 47a, 499 (2002).

[5] J. Amrit and M. X. François, J. Low Temp. Phys. 119, 27 (2000).

[6] J. Amrit and Q. Li, Adv. Cryog. Eng. 53a, 135 (2007); J. Amrit, J. Phys. D 39, 4472 (2006).

[7] W. Wasserbäch, Philos. Mag. A 38, 401 (1978).

[8] W. Wasserbäch, Phys. Status Solidi 84, 205 (1977).

[9] W. Wasserbäch, S. Sahling, R. O. Pohl, and E. Thompson, J. Low Temp. Phys. 127, 121 (2002).

[10] A. Connolly and K. Mendelssohn, Proc. R. Soc. 266, 429 (1962).

[11] A. C. Anderson and S. C. Smith, J. Phys. Chem. Solids 34, 111 (1973); A.C. Anderson and S. G. O'Hara, J. Low Temp. Phys. 15, 323 (1974).

[12] J. Amrit and M. X. François, J. Low Temp. Phys. 128, 113 (2002).

[13] P. Kneisel, G. Ciovati, W. Singer, X. Singer, D. Reschke, and A. Brinkman, in Proceedings of the 11th European Particle Accelerator Conference, Genoa, 2008 (EPS-AG, Genoa, Italy, 2008), p. 877.

[14] W. Singer, X. Singer, and P. Kneisel, AIP Conf. Proc. 927, 133 (2007).

[15] A. Ermakov, I. Jelezov, X. Singer, W. Singer, G. B. Viswanathan, V. Levit, H. L. Fraser, H. Wen, and M. Spiwek, J. Phys. Conf. Ser. 97, 012014 (2008).

[16] C. A. Cooper, A. Wu, P. Bauer, and C. Antoine, IEEE Trans. Appl. Supercond. 19, 1399 (2009).

[17] H. Padamsee, J. Knobloch, and T. Hays, $R F$ Superconductivity for Accelerators (John Wiley \& Sons, Inc., New York, 1998), Chap. 3 and 11.

[18] W. Schlump, H. Freyhardt, and E. Nembach, Acta Metall. 20, 257 (1972).

[19] A. Aizaz, P. Bauer, T. L. Grimm, N. T. Wright, and C.Z. Antoine, IEEE Trans. Appl. Supercond. 17, 1310 (2007).

[20] H. Safa, D. Moffat, B. Bonin, and F. Koechlin, J. Alloys Compd. 232, 281 (1996); also see Padamsee, Knobloch, and Hays, RF Superconductivity for Accelerators (Ref. [17]), Chap. 11.8.

[21] J. L. Challis, J. Phys. C 7, 481 (1974). 